\title{
Consequences and Limits of Nonlocal Strategies
}

\author{
Richard Cleve* $\quad$ Peter Høyer $^{\dagger} \quad$ Ben Toner $^{\ddagger} \quad$ John Watrous ${ }^{\S}$ \\ January 11, 2010
}

\begin{abstract}
This paper investigates the powers and limitations of quantum entanglement in the context of cooperative games of incomplete information. We give several examples of such nonlocal games where strategies that make use of entanglement outperform all possible classical strategies. One implication of these examples is that entanglement can profoundly affect the soundness property of two-prover interactive proof systems. We then establish limits on the probability with which strategies making use of entanglement can win restricted types of nonlocal games. These upper bounds may be regarded as generalizations of Tsirelson-type inequalities, which place bounds on the extent to which quantum information can allow for the violation of Bell inequalities. We also investigate the amount of entanglement required by optimal and nearly optimal quantum strategies for some games.
\end{abstract}

\section{Introduction}

This paper studies the implications of quantum entanglement to nonlocal games, which are cooperative games of incomplete information. There are two cooperating players in a nonlocal game: Alice and Bob. A referee, also sometimes called a verifier in this context, determines the game as follows. First, the referee randomly chooses questions for the players, drawn from finite sets according to some fixed probability distribution, and sends each player their question. Without communicating, and therefore without knowing (in general) what question was asked of the other player, Alice and Bob must each respond to the referee with an answer. The referee then evaluates some predicate on the questions asked of the players together with their answers, to determine whether they win or lose.

When analyzing a given nonlocal game, we assume that Alice and Bob have complete knowledge of the probability distribution that determines the referee's questions, as well as the predicate that determines whether they win or lose. Based on this information they can agree before the game starts on a joint strategy. A quantum strategy allows Alice and Bob to make use of a shared entangled state, while a classical strategy does not. For some nonlocal games, quantum strategies can allow the players to win with a higher probability than is possible for any classical strategy, due to the fact that measurements of an entangled state can result in correlations that are not achievable without the use of entanglement.

\footnotetext{
*Institute for Quantum Computing and School of Computer Science, University of Waterloo, Canada

${ }^{\dagger}$ Institute for Quantum Information Science and Department of Computer Science, University of Calgary, Canada

$\ddagger_{B Q P}$ Solutions Pty Ltd, Elsternwick, Australia

§Institute for Quantum Computing and School of Computer Science, University of Waterloo, Canada
} 
Nonlocal games are natural abstractions of multi-prover interactive proof systems (consisting of a single round of interaction with two provers), and have previously been considered in this context in purely classical terms [19, 13, 45]. They also provide a natural setting in which the quantum-physical notion of nonlocality can be cast. In quantum information theory, a Bell inequality is analogous to an upper bound on the probability with which Alice and Bob can win a nonlocal game using a classical strategy, and a violation of a Bell inequality is analogous to the situation in which a quantum strategy wins a particular nonlocal game with a higher probability than is possible for any classical strategy. Tsirelson-type inequalities are bounds on the amount by which quantum information can allow for the violation of Bell inequalities, and therefore are analogous to upper bounds on the probabilities with which nonlocal games can be won using quantum strategies.

We will begin our study of nonlocal games in Section 2, where we give precise definitions of nonlocal games and the classical and quantum values of these games. These values represent the optimal probabilities with which Alice and Bob can win the games using classical and quantum strategies, respectively.

Then, in Section 3, we present four examples of nonlocal games for which quantum strategies outperform classical strategies, including nonlocal games for which there exist perfect quantum strategies (meaning that the strategies win with probability one), but for which there do not exist perfect classical strategies. These examples are not new, but for the most part have been presented in the theoretical physics literature as hypothetical thought experiments, and their connections with nonlocal games and multi-prover interactive proofs are obscure. The simplicity of some of our presentations may help to elucidate some of the features of nonlocality.

Next, in Section 4, we discuss the implications of quantum strategies for nonlocal games to the study of multi-prover interactive proof systems. In particular, we exhibit two natural examples of two-prover interactive proof systems that are classically sound, but become unsound when the provers may employ quantum strategies.

Finally, in Section 5, we provide the beginnings of a systematic understanding of the limits of nonlocal strategies for two restricted classes of games: binary games and XOR games. Binary games are nonlocal games in which Alice and Bob each respond with a single bit, and XOR games are binary games for which the referee's predicate that determines whether Alice and Bob win or lose depends only on the parity (or exclusive-OR) of their answer bits. The results proved in this section include generalizations of Tsirelson's inequality. We also prove upper bounds on the amount of entanglement needed to play XOR games optimally or nearly optimally.

\section{Remark on a previous version of this paper}

A preliminary version of this paper appeared at the IEEE Conference on Computational Complexity in 2004, and was posted to the arXiv.org e-print server in April of 2004. This preliminary version contains an error: one of the technical lemmas (numbered Lemma 5.4 in the proceedings version and Lemma 5.5 in the arXiv.org version) is false. The present version corrects this mistake, which requires a new proof of Theorem 3 below.

Aside from this correction, the present version of this paper is similar to the preliminary version. While some revisions to the writing, notation, and organization of the paper have been made, and a few other minor points have been added, there is (for the most part) no discussion of newer work that was done subsequent to the writing of the preliminary version. (Readers interested in further developments on nonlocal games may, for instance, consult [17, 29, 32, 33].) 


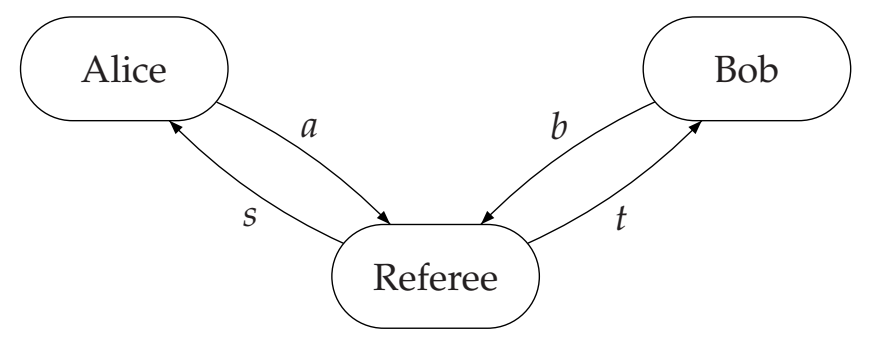

Figure 1: The communication structure of a nonlocal game.

\section{Definitions}

\section{Nonlocal games}

Let $\pi$ be a probability distribution on $S \times T$, and let $V$ be a predicate on $S \times T \times A \times B$, for finite, non-empty sets $S, T, A$, and $B$. Then $V$ and $\pi$ define a nonlocal game $G=G(V, \pi)$ as follows. A pair of questions $(s, t) \in S \times T$ is randomly chosen according to the distribution $\pi$, and $s$ is sent to Alice and $t$ is sent to Bob. Alice must respond with an answer $a \in A$ and Bob with an answer $b \in B$. Alice and Bob are not permitted to communicate after receiving $s$ and $t$, but they may agree on whatever sort of strategy they like prior to receiving their questions. They win if $V$ evaluates to 1 on $(s, t, a, b)$ and lose otherwise. To stress the fact that $(a, b)$ is correct or incorrect given questions $(s, t)$ we will denote the value of the predicate $V$ on $(s, t, a, b)$ as $V(a, b \mid s, t)$.

\section{Classical strategies and classical values of nonlocal games}

The classical value of a game $G$ is the maximum probability with which Alice and Bob can win $G$, ranging over all purely classical strategies. The classical value of a game $G$ will be denoted $\omega_{c}(G)$. A deterministic strategy is a restricted type of classical strategy in which $a$ and $b$ are simply functions of $s$ and $t$, respectively. The classical value of a game is always obtained by some deterministic strategy given that any probabilistic strategy can be expressed as a convex combination of deterministic strategies. In other words, it holds that

$$
\omega_{c}(G(V, \pi))=\max _{a, b} \sum_{s, t} \pi(s, t) V(a(s), b(t) \mid s, t),
$$

where the maximum is over all functions $a: S \rightarrow A$ and $b: T \rightarrow B$.

\section{Quantum strategies and quantum values of nonlocal games}

We will assume for this discussion and throughout the rest of the paper that the reader is familiar with the basics of quantum information, which is discussed (for instance) in the books [43], [35], and [31].

A quantum strategy for a game $G$ consists of an initial bipartite state $|\psi\rangle$ shared by Alice and Bob, a quantum measurement for Alice for each $s \in S$, and a quantum measurement for Bob for each $t \in T$. On input $(s, t)$, Alice performs her measurement corresponding to $s$ on her portion of $|\psi\rangle$, yielding an outcome $a$. Similarly, Bob performs his measurement corresponding to $t$ on his portion of $|\psi\rangle$, yielding outcome $b$. The results $a$ and $b$ are sent back to the referee. 
In more descriptive mathematical terms, a quantum strategy is determined by the following items.

1. A positive integer $n$ and a unit vector $|\psi\rangle \in \mathcal{A} \otimes \mathcal{B}$, for $\mathcal{A}$ and $\mathcal{B}$ isomorphic copies of the vector space $\mathbb{C}^{n}$. The space $\mathcal{A}$ represents Alice's part of $|\psi\rangle$ and the space $\mathcal{B}$ represents Bob's part.

2. Two collections of positive semidefinite $n \times n$ matrices

$$
\left\{X_{s}^{a}: s \in S, a \in A\right\} \quad \text { and } \quad\left\{Y_{t}^{b}: t \in T, b \in B\right\}
$$

satisfying

$$
\sum_{a \in A} X_{s}^{a}=\mathbb{1} \text { and } \sum_{b \in B} Y_{t}^{b}=\mathbb{1}
$$

for every choice of $s \in S$ and $t \in T$, where $\mathbb{1}$ denotes the $n \times n$ identity matrix. For each $s \in S$, the collection $\left\{X_{s}^{a}: a \in A\right\}$ describes the measurement performed by Alice on her part of $|\psi\rangle$ when she receives the question $s$. Likewise, for each $t \in T$, the collection $\left\{Y_{t}^{b}: b \in B\right\}$ describes Bob's measurement given the question $t$.

Given a question $s \in S$ for Alice and a question $t \in T$ for Bob, such a strategy causes Alice to answer with $a \in A$ and Bob to answer with $b \in B$ with probability $\left\langle\psi\left|X_{s}^{a} \otimes Y_{t}^{b}\right| \psi\right\rangle$. The probability that Alice and Bob win the game $G$ using such a strategy is therefore given by

$$
\sum_{(s, t) \in S \times T} \pi(s, t) \sum_{(a, b) \in A \times B}\left\langle\psi\left|X_{s}^{a} \otimes Y_{t}^{b}\right| \psi\right\rangle V(a, b \mid s, t) .
$$

The quantum value of a game $G$, denoted $\omega_{q}(G)$, is the supremum of the winning probabilities over all quantum strategies of Alice and Bob. It is not known if the quantum value is always achieved by some strategy, due to the fact that the number $n$ is not a priori bounded. For instance, it has not been ruled out that there could sometimes exist a sequence of strategies requiring increasing values of $n$ that win a game $G$ with probabilities converging to, but never reaching, its quantum value. (As is shown in [40], this phenomenon indeed occurs in an extended form of nonlocal games in which the referee can send, receive, and process quantum information.)

\section{Observables}

When we refer to an observable in this paper, we are referring to a Hermitian matrix that one associates with a projective measurement whose measurement outcomes are real numbers. More precisely, suppose that we have a measurement described by a collection of projection matrices $\left\{\Pi_{1}, \ldots, \Pi_{k}\right\}$ for which $\Pi_{1}+\cdots+\Pi_{k}=\mathbb{1}$, and suppose further that we associate the outcomes of the measurement with a collection of real numbers $\left\{\lambda_{1}, \ldots, \lambda_{k}\right\}$. Then the observable corresponding to this measurement is given by

$$
A=\sum_{j=1}^{k} \lambda_{j} \Pi_{j} .
$$

Given such a matrix $A$, one may determine the corresponding projective measurement by computing the spectral decomposition of $A$.

We will primarily use observables when discussing strategies for games that require Alice and Bob to perform two-outcome measurements, especially in the case of binary games (which are the main topic of Section (5). In this setting it is convenient to associate real numbers in the set 
$\{+1,-1\}$ with the binary values $\{0,1\}$ using the correspondence $0 \mapsto+1$ and $1 \mapsto-1$ (as is common when using discrete Fourier analysis of Boolean functions). When using this convention, the observable $A$ corresponding to a binary-valued projective measurement $\left\{\Pi_{0}, \Pi_{1}\right\}$ is given by $A=\Pi_{0}-\Pi_{1}$.

\section{Examples of nonlocal games}

The fact that entanglement can cause non-classical correlations is a familiar idea in quantum physics, introduced in a seminal 1964 paper by Bell [5]. In the following subsections, we give four examples of this phenomenon.

\subsection{The CHSH game}

Our first example of a game for which a quantum strategy outperforms any classical strategy is a well-known example based on the CHSH inequality, named for its discoverers Clauser, Horne, Shimony, and Holt [14]. Rephrased in terms of nonlocal games, the example is as follows. Let $S=T=A=B=\{0,1\}$, let $\pi$ be the uniform distribution on $S \times T$, and let $V$ be the predicate

$$
V(a, b \mid s, t)= \begin{cases}1 & \text { if } a \oplus b=s \wedge t \\ 0 & \text { otherwise }\end{cases}
$$

The classical value of the game $G=G(V, \pi)$ is $\omega_{c}(G)=3 / 4$, which is easily verified by considering all deterministic strategies. Using a quantum strategy, however, Alice and Bob can win this game with probability $\cos ^{2}(\pi / 8) \approx 0.85$. This probability is optimal, so we have $\omega_{q}(G)=$ $\cos ^{2}(\pi / 8)$. A description of a quantum strategy that achieves this probability of success follows, and the fact that it is optimal follows from Tsirelson's Inequality [34, 47].

First, let the entangled state shared by Alice and Bob be $|\psi\rangle=(|00\rangle+|11\rangle) / \sqrt{2}$, define

$$
\begin{aligned}
& \left|\phi_{0}(\theta)\right\rangle=\cos (\theta)|0\rangle+\sin (\theta)|1\rangle, \\
& \left|\phi_{1}(\theta)\right\rangle=-\sin (\theta)|0\rangle+\cos (\theta)|1\rangle,
\end{aligned}
$$

and let Alice and Bob's measurements be given as

$$
\begin{aligned}
& X_{0}^{a}=\left|\phi_{a}(0)\right\rangle\left\langle\phi_{a}(0)\right|, \\
& X_{1}^{a}=\left|\phi_{a}(\pi / 4)\right\rangle\left\langle\phi_{a}(\pi / 4)\right|, \\
& Y_{0}^{b}=\left|\phi_{b}(\pi / 8)\right\rangle\left\langle\phi_{b}(\pi / 8)\right|, \\
& Y_{1}^{b}=\left|\phi_{b}(-\pi / 8)\right\rangle\left\langle\phi_{b}(-\pi / 8)\right|
\end{aligned}
$$

for $a, b \in\{0,1\}$. Each of these matrices is a rank-one projection matrix, so the measurements Alice and Bob are making are examples of projective measurements. Given our particular choice of $|\psi\rangle$, we have $\langle\psi|X \otimes Y| \psi\rangle=\frac{1}{2} \operatorname{Tr}\left(X^{\top} Y\right)$ for arbitrary matrices $X$ and $Y$. Thus, as each of the matrices $X_{s}^{a}$ and $Y_{t}^{b}$ is real and symmetric, the probability that Alice and Bob answer $(s, t)$ with $(a, b)$ is $\frac{1}{2} \operatorname{Tr}\left(X_{s}^{a} Y_{t}^{b}\right)$. It is now routine to check that in every case, the correct answer is given with probability $\cos ^{2}(\pi / 8)$ and the incorrect answer with probability $\sin ^{2}(\pi / 8)$. 


\subsection{The Odd Cycle game}

For the following game, imagine that Alice and Bob are trying to convince the referee that an odd cycle of length $n$ is 2-colorable (which it is not, as $n$ is odd). The referee sends the name of a vertex to each of Alice and Bob such that the two vertices are either the same or adjacent. Alice and Bob each send one of two colors back to the referee. The referee's requirement is that, when the vertices are the same, the two colors should agree, and when the vertices are adjacent, the colors should be different.

Formally, let $n \geq 3$ be an odd integer, let $S=T=\mathbb{Z}_{n}$, and let $A=B=\{0,1\}$. Take $\pi$ to be the uniform distribution over the set $\left\{(s, t) \in \mathbb{Z}_{n} \times \mathbb{Z}_{n}: s=t\right.$ or $\left.s+1 \equiv t(\bmod n)\right\}$ and let $V$ be defined as

$$
V(a, b \mid s, t)= \begin{cases}1 & \text { if } a \oplus b=[s+1 \equiv t(\bmod n)] \\ 0 & \text { otherwise. }\end{cases}
$$

(Here we have written $[s+1 \equiv t(\bmod n)]$ to denote the Boolean value representing the truth or falsehood of the congruence $s+1 \equiv t(\bmod n)$.) This is a variation on a game based on the chained Bell inequalities of Braunstein and Caves [10] that generalize the CHSH inequality. It is also discussed by Vaidman [49].

It is easy to see that $\omega_{c}(G)=1-1 / 2 n$ for this game. Any deterministic strategy must fail for at least one of the possible pairs $(s, t)$, as an odd cycle cannot be 2-colored, while a strategy achieving success probability $1-1 / 2 n$ is that Alice and Bob let $a=s \bmod 2$ and $b=t \bmod 2$.

On the other hand, a quantum strategy can attain a success probability quadratically closer to 1 . The following quantum strategy [10] wins with probability $\cos ^{2}(\pi / 4 n) \geq 1-(\pi / 4 n)^{2}$. The shared state is the same as for the CHSH game:

$$
|\psi\rangle=\frac{1}{\sqrt{2}}(|00\rangle+|11\rangle)
$$

This time we define

$$
\begin{aligned}
& X_{s}^{a}=\left|\phi_{a}\left(\alpha_{s}\right)\right\rangle\left\langle\phi_{a}\left(\alpha_{s}\right)\right|, \\
& Y_{t}^{b}=\left|\phi_{b}\left(\beta_{t}\right)\right\rangle\left\langle\phi_{b}\left(\beta_{t}\right)\right|,
\end{aligned}
$$

where

$$
\begin{aligned}
& \alpha_{s}=\left(\frac{\pi}{2}-\frac{\pi}{2 n}\right) s+\frac{\pi}{4 n}, \\
& \beta_{t}=\left(\frac{\pi}{2}-\frac{\pi}{2 n}\right) t,
\end{aligned}
$$

and where $\left|\phi_{0}(\theta)\right\rangle$ and $\left|\phi_{1}(\theta)\right\rangle$ are as defined for the $\mathrm{CHSH}$ game. Given questions $(s, t)$, the probability that Alice and Bob answer the same bit may be calculated to be $\cos ^{2}\left(\alpha_{s}-\beta_{t}\right)$, which implies they answer different bits with probability $\sin ^{2}\left(\alpha_{s}-\beta_{t}\right)$. In case $s=t$ we have

$$
\alpha_{s}-\beta_{t}=\frac{\pi}{4 n}
$$

so they answer correctly (i.e., with $a=b)$ with probability $\cos ^{2}(\pi / 4 n)$. If $s+1 \equiv t(\bmod n)$, on the other hand, we have

$$
\alpha_{s}-\beta_{t}=\frac{\pi}{2}-\frac{\pi}{4 n}
$$

so they answer correctly (i.e., with $a \neq b$ ) with probability $\sin ^{2}(\pi / 2-\pi / 4 n)=\cos ^{2}(\pi / 4 n)$. Therefore this strategy answers correctly with probability $\cos ^{2}(\pi / 4 n)$ on every pair of questions. This quantum strategy is optimal, as we shall show in Corollary 9 later in the paper. 


\subsection{The Magic Square game}

The next game we consider is based on the fact that there does not exist a $3 \times 3$ binary matrix with the property that each row has even parity and each column has odd parity. It is a slight variation of an example presented by Aravind [3], which builds on work by Mermin [41, 42]. The idea is to ask Alice to fill in the values in either a row or a column of the matrix (randomly selected) and to ask Bob to fill in a single entry of the matrix, that is randomly chosen among the three entries given to Alice. The requirement is that the parity conditions are met by Alice's answers (even for rows, odd for columns) and that Bob's answer is consistent with Alice's answers.

Formally, let $S=\mathbb{Z}_{6}$ index the six possible questions to Alice (three rows plus three columns) and let $T=\mathbb{Z}_{9}$ index the nine possible questions to Bob (one for each entry of the matrix). Let $A=\{0,1\}^{3}$ and $B=\{0,1\}$. The predicate $V(a, b \mid s, t)$ is defined to take value 1 if and only if $a$ has the appropriate parity ( 0 for a row and 1 for a column) and the entry of $a$ corresponding to $t$ has value $b$. The distribution $\pi$ is the uniform distribution over $\{(s, t) \in S \times T$ : entry $t$ is in triple $s\}$. It is not hard to see that $\omega_{c}(G)=17 / 18$ for this game. It should be noted that, although it is convenient to set $A=\{0,1\}^{3}$ for this game, we could take $A=\{0,1\}^{2}$, because the third bit of Alice's output is determined by the first two bits and the parity constraints.

Remarkably, there exists a perfect quantum strategy for this game, meaning that the strategy wins with probability 1 . The particular strategy we will describe is derived from [3], where a slight variant of this game is presented.

This strategy is best described using the notion of observables, which were discussed at the end of Section 2. Consider the following $3 \times 3$ matrix of observables, each corresponding to a measurement of two qubits:

$$
\left(\begin{array}{ccc}
\sigma_{x} \otimes \sigma_{y} & \sigma_{y} \otimes \sigma_{x} & \sigma_{z} \otimes \sigma_{z} \\
\sigma_{y} \otimes \sigma_{z} & \sigma_{z} \otimes \sigma_{y} & \sigma_{x} \otimes \sigma_{x} \\
\sigma_{z} \otimes \sigma_{x} & \sigma_{x} \otimes \sigma_{z} & \sigma_{y} \otimes \sigma_{y}
\end{array}\right) .
$$

Here, the matrices $\sigma_{x}, \sigma_{y}$, and $\sigma_{z}$ are the (non-identity) Pauli matrices:

$$
\sigma_{x}=\left(\begin{array}{cc}
0 & 1 \\
1 & 0
\end{array}\right), \quad \sigma_{y}=\left(\begin{array}{cc}
0 & -i \\
i & 0
\end{array}\right), \quad \sigma_{z}=\left(\begin{array}{cc}
1 & 0 \\
0 & -1
\end{array}\right)
$$

Alice and Bob will share two copies of the state

$$
\left|\psi^{-}\right\rangle=\frac{1}{\sqrt{2}}|01\rangle-\frac{1}{\sqrt{2}}|10\rangle .
$$

To answer any question they receive about the $3 \times 3$ square, Alice and Bob simply measure their parts of the two shared states with respect to the corresponding observables in the above matrix. For example, if Alice is asked to assign values to the first row of the square, she measures her two qubits with respect to the observable $\sigma_{x} \otimes \sigma_{y}$, then with respect to the observable $\sigma_{y} \otimes \sigma_{x}$, and finally with respect to $\sigma_{z} \otimes \sigma_{z}$, each time obtaining a \pm 1 outcome corresponding to a binary value. Similarly, if Bob is asked to assign a value to the $(1,2)$ entry of the square, he measures his two qubits with respect to the observable $\sigma_{y} \otimes \sigma_{x}$.

To understand why this strategy works perfectly, a few basic observations are required. The first observation is that $\left|\psi^{-}\right\rangle$is a -1 eigenvector of each of the operators $\sigma_{x} \otimes \sigma_{x}, \sigma_{y} \otimes \sigma_{y}$, and $\sigma_{z} \otimes \sigma_{z}$, which implies

$$
\left\langle\psi^{-}\left|\sigma_{x} \otimes \sigma_{x}\right| \psi^{-}\right\rangle=\left\langle\psi^{-}\left|\sigma_{y} \otimes \sigma_{y}\right| \psi^{-}\right\rangle=\left\langle\psi^{-}\left|\sigma_{z} \otimes \sigma_{z}\right| \psi^{-}\right\rangle=-1 .
$$


This implies that Alice and Bob's answers are always in agreement, as the product of their measurement outcomes (considered as \pm 1 values) always equals 1 . For instance, if Alice and Bob both measure their two copies of $\left|\psi^{-}\right\rangle$with respect to the observable $\sigma_{y} \otimes \sigma_{x}$, the product of their outcomes will be

$$
\left\langle\psi^{-}\left|\sigma_{y} \otimes \sigma_{y}\right| \psi^{-}\right\rangle\left\langle\psi^{-}\left|\sigma_{x} \otimes \sigma_{x}\right| \psi^{-}\right\rangle=(-1)(-1)=1 .
$$

The second observation is that the Pauli matrices anti-commute in pairs:

$$
\sigma_{x} \sigma_{y}=-\sigma_{y} \sigma_{x}, \quad \sigma_{x} \sigma_{z}=-\sigma_{z} \sigma_{x}, \quad \sigma_{y} \sigma_{z}=-\sigma_{z} \sigma_{y} .
$$

This implies that the observables commute within any row or column. For instance, $\sigma_{y} \otimes \sigma_{x}$ and $\sigma_{z} \otimes \sigma_{z}$ both belong to the first row, and we have

$$
\left(\sigma_{y} \otimes \sigma_{x}\right)\left(\sigma_{z} \otimes \sigma_{z}\right)=\left(\sigma_{y} \sigma_{z}\right) \otimes\left(\sigma_{x} \sigma_{z}\right)=\left(-\sigma_{z} \sigma_{y}\right) \otimes\left(-\sigma_{z} \sigma_{x}\right)=\left(\sigma_{z} \sigma_{y}\right) \otimes\left(\sigma_{z} \sigma_{x}\right)=\left(\sigma_{z} \otimes \sigma_{z}\right)\left(\sigma_{y} \otimes \sigma_{x}\right) .
$$

This implies that Alice can simultaneously measure the three observables within whichever row or column she was asked-or equivalently that the outcomes she obtains do not depend on the order in which she performs these measurements.

The final observation that is required is that the product of the observables in each row is equal to $\mathbb{1} \otimes \mathbb{1}$, while the product of the observables in each column is $-\mathbb{1} \otimes \mathbb{1}$. This implies that Alice's parity requirements are always met.

\subsection{The Kochen-Specker game}

This game is based on the Kochen-Specker Theorem, which can be stated as follows.

Theorem 1 (Kochen and Specker [37]). There exists an explicit set of vectors $\left\{\left|v_{0}\right\rangle, \ldots,\left|v_{m-1}\right\rangle\right\}$ in $\mathbb{R}^{3}$ that cannot be $\{0,1\}$-colored so that both of the following conditions hold:

1. For every orthogonal pair of vectors $\left|v_{i}\right\rangle$ and $\left|v_{j}\right\rangle$, they are not both colored 1.

2. For every mutually orthogonal triple of vectors $\left|v_{i}\right\rangle,\left|v_{j}\right\rangle$, and $\left|v_{k}\right\rangle$, at least one of them is colored 1 .

The original theorem in [37] used 117 vectors, but this has subsequently been reduced to 31 vectors [44]. We will assume that every orthogonal pair of vectors in the set is part of an orthogonal triple-which is easily achieved by adding a few more vectors to the set-and that the vectors are normalized. Connections between the Kochen-Specker Theorem and nonlocality have previously been made in [28].

The Kochen-Specker game is defined relative to the above set of vectors. Alice receives a random triple of orthogonal vectors as her input and Bob receives a single vector randomly chosen from the triple as his input. Alice outputs a trit indicating which of her three vectors is assigned color 1 (implicitly, the other two vectors are assigned color 0 ). Bob outputs a bit assigning a color to his vector. The requirement is that Alice and Bob assign the same color to the vector that they receive in common.

It is straightforward to show that the existence of a perfect classical strategy for this game would violate the Kochen-Specker Theorem, so $\omega_{c}(G)<1$ for this game. On the other hand there is a perfect quantum strategy, using entanglement $|\psi\rangle=(|00\rangle+|11\rangle+|22\rangle) / \sqrt{3}$. Alice's projectors (for input $\left|v_{i}\right\rangle,\left|v_{j}\right\rangle,\left|v_{k}\right\rangle$ ) are $\left|v_{i}\right\rangle\left\langle v_{i}|,| v_{j}\right\rangle\left\langle v_{j}|,| v_{k}\right\rangle\left\langle v_{k}\right|$, and Bob's projectors (for input $\left.\left|v_{l}\right\rangle\right)$ are $\left|v_{l}\right\rangle\left\langle v_{l}\right|$ and $\mathbb{1}-\left|v_{l}\right\rangle\left\langle v_{l}\right|$. 


\section{Connections with multi-prover interactive proof systems}

The two-prover interactive proof system model was defined by Ben-Or, Goldwasser, Kilian, and Wigderson [7], and has been the focus of a great deal of study. Babai, Fortnow, and Lund [4] proved that every language in NEXP has a two-prover interactive proof system. Several refinements to this result were made [12, 18, 39], leading to a proof by Feige and Lovász [19] that a language is in NEXP if and only if it has a two-prover one-round proof system with perfect completeness and exponentially small soundness error.

In most work on multi-prover interactive proof systems, the provers are computationally unbounded, subject to the restriction that they cannot communicate with each other during the course of the protocol. Because the spirit of the interactive proof system paradigm is to bound the capabilities of the verifier rather than the prover(s), it is natural to consider prover strategies that entail sharing entangled quantum information prior to the execution of the proof system. Note that such a strategy does not necessarily require the computationally bounded verifier to manipulate (or know anything about) quantum information. However, much of the study of multi-prover interactive proof systems occurred prior to the mid 1990s, when quantum information was not well-known within the theoretical computer science community, and quantum strategies were generally not considered. In fact, the methodologies for analyzing these proof systems usually make the implicit assumption that provers are restricted to classical strategies.

In this section, we consider what happens when the provers can employ quantum strategies. We do not make any change to the verifier, who remains classical, and all communication between the verifier and the provers remains classical 1 A natural question is: What is the expressive power of such proof systems?

Let us write MIP and MIP* to distinguish between the cases of no shared entanglement and shared entanglement, respectively. That is, MIP denotes the class of languages recognized by multi-prover interactive proof systems where all communication between the provers and verifier is classical and the provers do not share entanglement (as has been implicitly assumed in previous contexts). The definition of $\mathrm{MIP}^{*}$ is identical to that of MIP, except that the provers may share an arbitrary entangled quantum state at the beginning of the protocol. Furthermore, let $\operatorname{MIP}[k]$ and $\mathrm{MIP}^{*}[k]$ denote the same classes, but with the number of provers set to $k$. It is known that $\mathrm{MIP}=\mathrm{MIP}[2]=\mathrm{NEXP}$. We do not know any relationships between MIP*, MIP*[2] and NEXP, except the trivial containment $\mathrm{MIP}^{*}[2] \subseteq \mathrm{MIP}^{*}$.

A one-round two-prover interactive proof system is one where the interaction is restricted to two stages: a query stage where the verifier sends information to the provers, and a response stage where the provers send information to the verifier. Note that such a proof system associates a nonlocal game $G_{x}$ to each string $x$ with the following property. For all yes-inputs $x$, the value of $\omega_{q}\left(G_{x}\right)$ is close to one, and, for all no-inputs $x$, the value of $\omega_{q}\left(G_{x}\right)$ is close to zero.

We give two examples of natural two-prover one-round proof systems that are classically sound, but become unsound when the provers use quantum strategies: one is for languages that express graph chromatic numbers and the other is for 3-SAT. These examples are related to the examples in Section 3. We also explain why the existing proofs that equate MIP with NEXP break down in terms of their methodology in the case of MIP*. Results in [36] imply that, if the amount of entanglement between the provers is polynomially bounded, then any language recognized by an MIP* proof system is contained in NEXP; but without this polynomial restriction, we do not know if this holds.

\footnotetext{
${ }^{1}$ Kobayashi and Matsumoto [36] consider a related but different model, where the provers and the verifier manipulate quantum information and quantum communication occurs between the verifier and the provers.
} 


\subsection{Graph Coloring proof system}

The Odd Cycle game in Section 3.2 can be regarded as a protocol where two provers are trying to convince a verifier that a particular graph is two-colorable. This idea generalizes to any graph $G$ and number of colors $k$. The verifier asks each prover for the color (among $k$ possibilities) of a vertex and requires that the colors be the same whenever each prover gets the same vertex and different whenever the provers get adjacent vertices. Formally, the game for $G$ and $k$ is as follows. Let $S=T=V(G)$, let $A=B=\mathbb{Z}_{k}$, let

$$
V(a, b \mid s, t)= \begin{cases}1 & \text { if }(s=t \text { and } a=b) \text { or }((s, t) \in E(G) \text { and } a \neq b) \\ 0 & \text { otherwise, }\end{cases}
$$

and $\pi$ be the uniform distribution on $\{(s, s): s \in V(G)\} \cup\{(s, t) \in E(G)\}$.

If $G$ is $k$-colorable then the provers can satisfy $V$ by basing their answers on a valid coloring of $G$. Therefore, the value of the associated game is 1 . If $G$ is not $k$-colorable then, for any classical strategy on the part of the provers, there must be an inconsistency for some value of $(s, t)$, so the classical value of the associated game is at most $1-1 /(|V(G)|+|E(G)|)$. The verifier can amplify the difference between the two cases ( $k$-colorable and not $k$-colorable) by repeating this game a polynomial number of times (in parallel [45]). Thus this is a classical two-prover interactive proof system for the language consisting of all $k$-colorable graphs.

This proof system breaks down in the case of entangled provers. Based on a protocol in [9], there exists a sequence of graphs $G_{n}$ (where $n$ ranges over all powers of two) with the following properties. First, for any $n$, there is a perfect quantum strategy for the Graph Coloring proof system with graph $G_{n}$ and $k=n$ colors. Second, for sufficiently large $n, G_{n}$ is not $n$-colorable.

For any $n, G_{n}$ is simple to describe: it has vertices $\{0,1\}^{n}$ and two vertices are adjacent if and only if the Hamming distance between them is $n / 2$. However, results in [9] show that there exists an $n$ such that $G_{n}$ is not $n$-colorable, without giving an explicit $n$ for which this holds. (The proof is based on a related result in [11], which makes use of a combinatorial result in [23].) The result is made explicit in [24], where it is shown that $G_{16}$ is not 16-colorable. Thus, the resulting graph for which the Graph Coloring proof system breaks down has $2^{16}$ vertices, and it can be simplified by taking only half of its vertices, resulting in a graph of 32,768 vertices.

\subsection{3-SAT proof system}

Next we consider a simple and natural two-prover interactive proof system for proving that 3-CNF formulas are satisfiable. The verifier sends the first prover (Alice) a clause and the second prover (Bob) a variable from that clause. Alice must provide a satisfying assignment to the variables in that clause and Bob must assign a value for the variable he receives that is consistent with Alice's assignment.

This proof system associates a nonlocal game $G_{f}$ with every 3-CNF Boolean formula $f$ over variables $x_{0}, \ldots, x_{n-1}$ with $m$ clauses $c_{0}, \ldots, c_{m-1}$. Specifically, we take

$$
S=\mathbb{Z}_{m}, \quad T=\mathbb{Z}_{n}, \quad A=\{0,1\}^{3}, \quad \text { and } \quad B=\{0,1\} .
$$

For each clause, every $a \in\{0,1\}^{3}$ induces an assignment to each variable that occurs in the clause in a natural way. The predicate $V(a, b \mid s, t)$ takes the value 1 if and only if the assignment for the variables in $c_{s}$ induced by $a$ satisfies $c_{s}$ and is consistent with the assignment $x_{t}=b$, and the distribution $\pi$ may be taken to be uniform on

$$
\left\{(s, t) \in S \times T \text { : clause } c_{s} \text { contains variable } x_{t}\right\} .
$$


If $f$ is satisfiable then $\omega_{c}\left(G_{f}\right)=1$ by the two provers returning values corresponding to a specific truth assignment. If $f$ is unsatisfiable then $\omega_{c}\left(G_{f}\right) \leq 1-1 / 3 m$, as then at least one of the $3 m$ possible $(s, t)$ queries must violate the predicate.

However, this proof system breaks down in the case of entangled provers. Upon seeing the aforementioned counterexample for the Graph Coloring proof system, Ambainis [2] showed that a counterexample for 3-SAT could be based on it. Intuitively, the idea is to construct a 3-CNF formula that, for truth assignment $x$, expresses the statement " $x$ is a $k$-coloring of $G$." Based on the above counterexample graph with 32,768 vertices (the smallest that we are aware of), the resulting 3-SAT formula consists of roughly $10^{8}$ clauses.

A simpler counterexample can be based on the Magic Square game in Section 3.3 that consists of 24 clauses. We will construct an instance of 3-SAT, where the resulting formula is not satisfiable but for which there is a perfect quantum strategy for the above two-prover proof system. Let the variables be $x_{00}, x_{01}, x_{02}, x_{10}, x_{11}, x_{12}, x_{20}, x_{21}, x_{22}$, which intuitively correspond to a $3 \times 3$ Boolean matrix. There are six parity conditions in the Magic Square game: each row has even parity and each column has odd parity. Each parity condition can be expressed with four clauses. For example, for the first row,

$$
\left(\bar{x}_{00} \vee \bar{x}_{01} \vee \bar{x}_{02}\right) \wedge\left(\bar{x}_{00} \vee x_{01} \vee x_{02}\right) \wedge\left(x_{00} \vee \bar{x}_{01} \vee x_{02}\right) \wedge\left(x_{00} \vee x_{01} \vee \bar{x}_{02}\right)
$$

is satisfied if and only if $x_{00} \oplus x_{01} \oplus x_{02}=0$. Thus 24 clauses suffice to express all six parity conditions. This formula is unsatisfiable, but the perfect quantum strategy for the Magic Square game in Section 3.3 defeats the 3-SAT game for this formula with certainty.

\subsection{Oracularization paradigm}

The above example also constitutes a counterexample to a commonly-used primitive that enables a two-prover system to simulate an oracle machine. An oracle machine is a one-prover interactive system where the prover's responses to a series of questions are required to be non-adaptive. Nonadaptive means that when the prover receives a series of queries $s_{1}, s_{2}, \ldots, s_{m}$, his response to $s_{i}$ must be a function of $s_{i}$ alone, not depending on any $s_{j}$ for $j \neq i$. There is a simple oracle machine proof system for 3-SAT, where a random clause is selected and its three variables are sent as three queries to the prover, who must return a value for each one. The verifier accepts if and only if the responses satisfy the clause. The prover's success probability is less than one whenever the formula is unsatisfiable.

Fortnow, Rompel, and Sipser [22] showed that, with a second prover, who is sent a single randomly chosen query from those of the first prover, the first prover must behave as an oracle or be detected with positive probability. Nevertheless, the above quantum strategy for the magic square game is a counterexample to this result for the case of entangled provers. Because this is a component in the proof that MIP $=\mathrm{NEXP}$, this proof does not carry over to the case of MIP*.

\section{Binary games and XOR games}

In this section we focus our attention on two simple types of games that we call binary games and XOR games. Binary games are games in which Alice and Bob's answers are bits: $A=B=\{0,1\}$. XOR games are binary games that are further restricted in that the value of the predicate $V$ may depend only on $a \oplus b$ and not on $a$ and $b$ independently. The CHSH and Odd Cycle games are examples of XOR games. 
We begin by establishing some basic properties of binary games and XOR games, and then prove upper bounds on the quantum values of these games. In particular, we prove that any binary game having a perfect quantum strategy also has a perfect classical strategy, and for XOR games we obtain quantitatively stronger upper bounds through the use of a theorem due to Tsirelson. We then prove upper bounds on the amount of entanglement required for Alice and Bob to play XOR games optimally and nearly optimally, and finally point out a further connection between these games and multi-prover interactive proof systems.

\subsection{Optimality of projective measurements for binary games}

In accordance with the description of quantum strategies in Section 2 , Alice and Bob's strategy for a binary game consists of a shared entangled state $|\psi\rangle \in \mathcal{A} \otimes \mathcal{B}$, for $\mathcal{A}$ and $\mathcal{B}$ isomorphic copies of the space $\mathbb{C}^{n}$, together with a collection of matrices representing measurements: $X_{s}^{0}$ and $X_{s}^{1}$, for each $s \in S$, and $Y_{t}^{0}$ and $Y_{t}^{1}$, for each $t \in T$. In this section we observe that these matrices may always be taken to be projection matrices, even when restricted to the support of the vector $|\psi\rangle$. This fact is easily proved, and in some sense it is more direct than the well-known fact that general measurements can be simulated by projective measurements. (Note also that this simulation requires the use of an ancillary system, and therefore does not immediately imply the claimed fact.)

Proposition 2. Let $G$ be a binary game, and let $|\psi\rangle \in \mathcal{A} \otimes \mathcal{B}$ be any fixed state shared by Alice and Bob. Then among the set of all strategies for $G$ for which the shared entangled state is $|\psi\rangle$, there is an optimal strategy for which all of Alice's measurements $\left\{X_{s}^{0}, X_{s}^{1}\right\}$ and Bob's measurements $\left\{Y_{t}^{0}, Y_{t}^{1}\right\}$ are projective measurements on $\mathcal{A}$ and $\mathcal{B}$, respectively.

Proof. Consider any choice of measurements $\left\{X_{s}^{0}, X_{s}^{1}\right\}$ and $\left\{Y_{t}^{0}, Y_{t}^{1}\right\}$. We will show that these measurements can be replaced by projective measurements on $\mathcal{A}$ and $\mathcal{B}$ without decreasing the probability that Alice and Bob win. Because the set of all possible choices for the measurements $\left\{X_{s}^{0}, X_{s}^{1}\right\}$ and $\left\{Y_{t}^{0}, Y_{t}^{1}\right\}$ can be represented as a compact set, the theorem then follows from the fact that there must exist an optimal choice of measurements.

For each choice of $s \in S$, let

$$
X_{s}^{0}=\sum_{j=1}^{n} \lambda_{s, j}\left|\phi_{s, j}\right\rangle\left\langle\phi_{s, j}\right|,
$$

be a spectral decomposition of $X_{s}^{0}$. We will consider the possible strategies for Alice that are obtained by viewing the vectors $\left\{\left|\phi_{s, j}\right\rangle: s \in S, j=1 \ldots, n\right\}$ as being fixed but the eigenvalues $\left\{\lambda_{s, j}: s \in S, j=1 \ldots, n\right\}$ as being variables. Because it must be the case that $0 \leq X_{s}^{0} \leq \mathbb{1}$, these eigenvalues are subject to the constraint that $\lambda_{s, j} \in[0,1]$ for all $s \in S$ and $j=1, \ldots, n$. Also note that because $X_{s}^{1}=\mathbb{1}-X_{s}^{0}$, it holds that

$$
X_{s}^{1}=\sum_{j=1}^{n}\left(1-\lambda_{s, j}\right)\left|\phi_{s, j}\right\rangle\left\langle\phi_{s, j}\right| .
$$

Now, the probability that Alice and Bob's strategy wins is

$$
\operatorname{Pr}[\text { Alice and Bob win }]=\sum_{s, t} \pi(s, t) \sum_{a, b} V(a, b \mid s, t)\left\langle\psi\left|X_{s}^{a} \otimes Y_{t}^{b}\right| \psi\right\rangle,
$$

which may be expressed as an affine function of the variables $\left\{\lambda_{s, j}: s \in S, j=1, \ldots, n\right\}$. Subject to the constraint that each $\lambda_{s, j} \in[0,1]$, this function must therefore be maximized for some choice 
for these variables for which $\lambda_{s, j} \in\{0,1\}$ for each $s \in S$ and $j=1, \ldots, n$. For such a choice of the eigenvalues $\lambda_{s, j}$ we have that each of Alice's measurements $\left\{X_{s}^{0}, X_{s}^{1}\right\}$ becomes a projective measurement. Repeating a similar argument for Bob's measurements yields the desired result.

\subsection{Perfect strategies for binary games}

We now prove that if $G$ is a binary game for which there exists a perfect quantum strategy, then $G$ has a perfect classical strategy as well: $\omega_{c}(G)=1$. This fact depends on the assumption that the game is binary. The Kochen-Specker game discussed in Section 2 , for instance, is a ternary-binary game (i.e., $A=\{0,1,2\}$ and $B=\{0,1\}$ ) for which there exists a perfect quantum strategy, but no perfect classical strategy.

Theorem 3. Let $G$ be a binary game. If there exists a quantum strategy for $G$ that wins with probability 1, then $\omega_{c}(G)=1$.

Proof. Assume that a perfect quantum strategy for $G$ is given. More specifically, we assume that this strategy uses a shared entangled state $|\psi\rangle \in \mathcal{A} \otimes \mathcal{B}$, for $\mathcal{A}$ and $\mathcal{B}$ isomorphic copies of $\mathbb{C}^{n}$, and by Proposition 2 we may assume that Alice and Bob's measurements are projective measurements on $\mathcal{A}$ and $\mathcal{B}$, respectively. It is therefore possible to describe these measurements by two collections of \pm 1 observables $\left\{A_{s}: s \in S\right\}$ and $\left\{B_{t}: t \in T\right\}$ on $\mathcal{A}$ and $\mathcal{B}$, respectively, and for the remainder of the proof we will consider for the sake of convenience that Alice and Bob's answer sets are given by $\{+1,-1\}$ rather than $\{0,1\}$. We note that the probability that Alice and Bob respond to a given question pair $(s, t) \in S \times T$ with the answers $(\alpha, \beta) \in\{+1,-1\} \times\{+1,-1\}$ is given by

$$
\begin{aligned}
q(\alpha, \beta \mid s, t) & =\frac{1}{4}\left\langle\psi\left|\left(\mathbb{1}+\alpha A_{s}\right) \otimes\left(\mathbb{1}+\beta B_{t}\right)\right| \psi\right\rangle \\
& =\frac{1}{4}+\frac{\alpha}{4}\left\langle\psi\left|A_{s} \otimes \mathbb{1}\right| \psi\right\rangle+\frac{\beta}{4}\left\langle\psi\left|\mathbb{1} \otimes B_{t}\right| \psi\right\rangle+\frac{\alpha \beta}{4}\left\langle\psi\left|A_{s} \otimes B_{t}\right| \psi\right\rangle .
\end{aligned}
$$

We will now define functions $a: S \rightarrow\{+1,-1\}$ and $b: T \rightarrow\{+1,-1\}$ that represent a perfect deterministic strategy for Alice and Bob. First, fix an orthonormal basis $\left\{\left|\phi_{1}\right\rangle, \ldots,\left|\phi_{n^{2}}\right\rangle\right\}$ of $\mathcal{A} \otimes \mathcal{B}$ such that $\left|\phi_{1}\right\rangle=|\psi\rangle$ and where $\left|\phi_{2}\right\rangle, \ldots,\left|\phi_{n^{2}}\right\rangle$ are chosen arbitrarily (subject to the constraint of orthonormality). Next, define functions $k: S \rightarrow\left\{1, \ldots, n^{2}\right\}$ and $\ell: T \rightarrow\left\{1, \ldots, n^{2}\right\}$ as

$$
\begin{aligned}
& k(s)=\min \left\{j \in\left\{1, \ldots, n^{2}\right\}:\left\langle\phi_{j}\left|A_{s} \otimes \mathbb{1}\right| \psi\right\rangle \neq 0\right\} \\
& \ell(t)=\min \left\{j \in\left\{1, \ldots, n^{2}\right\}:\left\langle\phi_{j}\left|\mathbb{1} \otimes B_{t}\right| \psi\right\rangle \neq 0\right\}
\end{aligned}
$$

and also define a function $\mathcal{K}: \mathbb{C} \backslash\{0\} \rightarrow\{+1,-1\}$ over the nonzero complex numbers as

$$
\kappa(z)= \begin{cases}+1 & \text { if } \arg (z) \in[0, \pi) \\ -1 & \text { if } \arg (z) \in[\pi, 2 \pi) .\end{cases}
$$

Finally, define

$$
a(s)=\kappa\left(\left\langle\phi_{k(s)}\left|A_{s} \otimes \mathbb{1}\right| \psi\right\rangle\right) \quad \text { and } \quad b(t)=\kappa\left(\left\langle\phi_{\ell(t)}\left|\mathbb{1} \otimes B_{t}\right| \psi\right\rangle\right) .
$$

We will now prove that the functions $a$ and $b$ define a perfect deterministic strategy for $G$, so that $\omega_{\mathcal{c}}(G)=1$. To do this, we prove that every question pair $(s, t)$ is answered with $(a(s), b(t))$ by the given quantum strategy with a positive probability: $q(a(s), b(t) \mid s, t)>0$. Given that the quantum strategy is perfect, it follows that $(a(s), b(t))$ is correct for $(s, t)$ whenever $\pi(s, t)>0$. 
To prove that $q(a(s), b(t) \mid s, t)>0$ for every choice of $s$ and $t$, we first observe the following two facts:

1. if $\left\langle\psi\left|A_{s} \otimes \mathbb{1}\right| \psi\right\rangle \neq 0$, then $a(s)\left\langle\psi\left|A_{s} \otimes \mathbb{1}\right| \psi\right\rangle>0$, and

2. if $\left\langle\psi\left|\mathbb{1} \otimes B_{t}\right| \psi\right\rangle \neq 0$, then $b(t)\left\langle\psi\left|\mathbb{1} \otimes B_{t}\right| \psi\right\rangle>0$.

The first fact follows from the observation that $\left\langle\psi\left|A_{s} \otimes \mathbb{1}\right| \psi\right\rangle$ is a real number, and if it is nonzero we have $k(s)=1$, so that $a(s)=\operatorname{sign}\left(\left\langle\psi\left|A_{s} \otimes \mathbb{1}\right| \psi\right\rangle\right)$. The second fact is similar to the first. It follows that if either or both of the numbers $\left\langle\psi\left|A_{s} \otimes \mathbb{1}\right| \psi\right\rangle$ or $\left\langle\psi\left|\mathbb{1} \otimes B_{t}\right| \psi\right\rangle$ is nonzero, then

$$
a(s)\left\langle\psi\left|A_{s} \otimes \mathbb{1}\right| \psi\right\rangle+b(t)\left\langle\psi\left|\mathbb{1} \otimes B_{t}\right| \psi\right\rangle>0 .
$$

As $\left\langle\psi\left|A_{s} \otimes B_{t}\right| \psi\right\rangle \geq-1$, it follows that

$$
q(a(s), b(t) \mid s, t)=\frac{1}{4}+\frac{a(s)}{4}\left\langle\psi\left|A_{s} \otimes \mathbb{1}\right| \psi\right\rangle+\frac{b(t)}{4}\left\langle\psi\left|\mathbb{1} \otimes B_{t}\right| \psi\right\rangle+\frac{a(s) b(t)}{4}\left\langle\psi\left|A_{s} \otimes B_{t}\right| \psi\right\rangle>0 .
$$

The quantum strategy therefore results in the answers $(a(s), b(t))$ to the question pair $(s, t)$ with a nonzero probability.

The remaining case to consider is that $\left\langle\psi\left|A_{s} \otimes \mathbb{1}\right| \psi\right\rangle=\left\langle\psi\left|\mathbb{1} \otimes B_{t}\right| \psi\right\rangle=0$. In this case we have

$$
q(a(s), b(t) \mid s, t)=\frac{1}{4}+\frac{a(s) b(t)}{4}\left\langle\psi\left|A_{s} \otimes B_{t}\right| \psi\right\rangle .
$$

As in the first case, we wish to prove that $q(a(s), b(t) \mid s, t)$ is positive, so we assume toward contradiction that $q(a(s), b(t) \mid s, t)=0$, which implies that $a(s) b(t)\left\langle\psi\left|A_{s} \otimes B_{t}\right| \psi\right\rangle=-1$. We have that

$$
a(s) b(t)\left\langle\psi\left|A_{s} \otimes B_{t}\right| \psi\right\rangle=\sum_{j=1}^{n^{2}} a(s) b(t)\left\langle\psi\left|A_{s} \otimes \mathbb{1}\right| \phi_{j}\right\rangle\left\langle\phi_{j}\left|\mathbb{1} \otimes B_{t}\right| \psi\right\rangle,
$$

and we observe that this quantity corresponds to the inner product of the two $n^{2}$ dimensional unit vectors whose $j$-th entries are given by $a(s)\left\langle\phi_{j}\left|A_{s} \otimes \mathbb{1}\right| \psi\right\rangle$ and $b(t)\left\langle\phi_{j}\left|\mathbb{1} \otimes B_{t}\right| \psi\right\rangle$, respectively. If it is the case that this inner product is -1 , then it must hold that

$$
a(s)\left\langle\phi_{j}\left|A_{s} \otimes \mathbb{1}\right| \psi\right\rangle=-b(t)\left\langle\phi_{j}\left|\mathbb{1} \otimes B_{t}\right| \psi\right\rangle
$$

for every choice of $j=1, \ldots, n^{2}$. However, if it is the case that $k(s) \neq \ell(t)$, then (4) must fail to hold for $j=\min \{k(s), \ell(t)\}$, while if $k(s)=\ell(t)$, then for $j=k(s)=\ell(t)$ we find that the values $\arg \left(a(s)\left\langle\phi_{j}\left|A_{s} \otimes \mathbb{1}\right| \psi\right\rangle\right)$ and $\arg \left(b(t)\left\langle\phi_{j}\left|\mathbb{1} \otimes B_{t}\right| \psi\right\rangle\right)$ are both in the range $[0, \pi)$, and therefore (4) again fails to hold. We have obtained a contradiction, which completes the proof.

\subsection{Bounds on values of XOR games}

We now prove upper bounds on quantum strategies for XOR games, which are binary games where the predicate $V(a, b \mid s, t)$ depends only on $c=a \oplus b$ and not $a$ and $b$ independently. It will be convenient to view the predicate $V$ as taking only three inputs in this case, so we write $V(a \oplus b \mid s, t)$ rather than $V(a, b \mid s, t)$. 


\section{Tsirelson's correspondence}

To establish upper bounds on quantum strategies for XOR games, we begin with a theorem due to Tsirelson [48]. This theorem is most naturally stated in terms of observables, as described in Section 2. (By Proposition 2, there will be no loss of generality in considering only projective measurements, or equivalently their corresponding observables, in the context of binary games.)

Theorem 4 (Tsirelson [48]). Let $S$ and $T$ be finite, nonempty sets, and let $\left\{c_{s, t}:(s, t) \in S \times T\right\}$ be a collection of real numbers in the range $[-1,1]$. Then the following are equivalent:

1. There exists a positive integer $n$, complex Hilbert spaces $\mathcal{A}$ and $\mathcal{B}$ with finite dimension $n$, a unit vector $|\psi\rangle \in \mathcal{A} \otimes \mathcal{B}$, a collection $\left\{A_{s}: s \in S\right\}$ of \pm 1 observables on $\mathcal{A}$, and a collection $\left\{B_{t}: t \in T\right\}$ of \pm 1 observables on $\mathcal{B}$, such that

$$
\left\langle\psi\left|A_{s} \otimes B_{t}\right| \psi\right\rangle=c_{s, t}
$$

for all $(s, t) \in S \times T$.

2. There exists a positive integer $m$ and two collections $\left\{\left|u_{s}\right\rangle: s \in S\right\}$ and $\left\{\left|v_{t}\right\rangle: t \in T\right\}$ of unit vectors in $\mathbb{R}^{m}$ such that

$$
\left\langle u_{s} \mid v_{t}\right\rangle=c_{s, t}
$$

for all $(s, t) \in S \times T$.

Moreover, if the first item holds for a fixed choice of $n$, then the second holds for $m=2 n^{2}$; and if the second item holds for a fixed choice of $m$, then the first holds for $n=2^{\lceil m / 2\rceil}$.

\section{Advantage over the trivial strategy}

Next, to state certain upper bounds on $\omega_{q}(G)$ for XOR games, it will be helpful to define the trivial random strategy for Alice and Bob as one where they ignore their inputs and answer uniformly generated random bits. If $\tau(G)$ denotes the success probability of game $(G, \pi)$ when Alice and Bob are restricted to this trivial strategy, then

$$
\tau(G)=\frac{1}{2} \sum_{c \in\{0,1\}} \sum_{s, t} \pi(s, t) V(c \mid s, t) .
$$

Proposition 5. Let $G(V, \pi)$ be an XOR game and let $m=\min (|S|,|T|)$. Then

$$
\omega_{q}(G)-\tau(G)=\frac{1}{2} \max _{\left\{\left|u_{s}\right\rangle\right\},\left\{\left|v_{t}\right\rangle\right\}} \sum_{s, t} \pi(s, t)(V(0 \mid s, t)-V(1 \mid s, t))\left\langle u_{s} \mid v_{t}\right\rangle,
$$

where the maximum is over all choices of unit vectors $\left\{\left|u_{s}\right\rangle: s \in S\right\} \cup\left\{\left|v_{t}\right\rangle: t \in T\right\}$ in $\mathbb{R}^{m}$.

Proof. Consider any quantum strategy for Alice and Bob given by a shared entangled state $|\psi\rangle \in$ $\mathcal{A} \otimes \mathcal{B}$ and collections of observables $\left\{A_{s}: s \in S\right\}$ and $\left\{B_{t}: t \in T\right\}$. We associate with each $A_{s}$ a real unit vector $\left|u_{s}\right\rangle$ and with each $B_{t}$ a real unit vector $\left|v_{t}\right\rangle$, according to Theorem 4. On input $(s, t)$, the probability that Alice and Bob's answers are equal is

$$
\left\langle\psi\left|X_{s}^{0} Y_{t}^{0}+X_{s}^{1} Y_{t}^{1}\right| \psi\right\rangle=\frac{1}{2}+\frac{1}{2}\left\langle\psi\left|A_{s} \otimes B_{t}\right| \psi\right\rangle=\frac{1}{2}+\frac{1}{2}\left\langle u_{s} \mid v_{t}\right\rangle
$$


It follows that their answers are not equal with probability $\left(1-\left\langle u_{s} \mid v_{t}\right\rangle\right) / 2$. Hence the probability that Alice and Bob win using this strategy is

$$
\frac{1}{2} \sum_{s, t, c} \pi(s, t) V(c \mid s, t)+\frac{1}{2} \sum_{s, t} \pi(s, t)(V(0 \mid s, t)-V(1 \mid s, t))\left\langle u_{s} \mid v_{t}\right\rangle
$$

Assuming the spaces $\mathcal{A}$ and $\mathcal{B}$ have dimension $n$, the vectors $\left|u_{s}\right\rangle$ and $\left|v_{t}\right\rangle$ are unit vectors in $\mathbb{R}^{2 n^{2}}$. Although the dimension $n$ is a priori unbounded, the winning probability depends only on the inner products among the unit vectors $\left\{\left|u_{s}\right\rangle: s \in S\right\}$ and $\left\{\left|v_{t}\right\rangle: t \in T\right\}$. We may therefore project onto the span of $\left\{\left|u_{s}\right\rangle: s \in S\right\} \cup\left\{\left|v_{t}\right\rangle: t \in T\right\}$, which is a space with dimension at most $|S|+|T|$. Indeed, it is sufficient to project the vectors $\left\{\left|u_{s}\right\rangle: s \in S\right\}$ onto the span of the vectors $\left\{\left|v_{t}\right\rangle: t \in T\right\}$ (or vice versa). The dimension of this space is at most $m=\min (|S|,|T|)$. Without loss of generality, let us assume $|S| \leq|T|$. Although the vectors $\left\{\left|u_{s}\right\rangle: s \in S\right\}$ will not necessarily remain unit vectors after orthogonal projection, the maximum over all vectors $\left\{\left|u_{s}\right\rangle \in \mathbb{R}^{|T|}: s \in S, \|\left|u_{s}\right\rangle \| \leq 1\right\}$ is achieved by points on the boundary-unit vectors-and so it is sufficient to restrict to this case.

We now show this strategy can be realized as a quantum protocol. The maximization in (5) is over a compact set, so the maximum is achieved by some choice of vectors $\left\{\left|u_{s}\right\rangle: s \in S\right\}$ and $\left\{\left|v_{t}\right\rangle: t \in T\right\}$ in $\mathbb{R}^{m}$. Let $|\psi\rangle$ be a maximally entangled state on $\lceil m / 2\rceil$ qubits. By Theorem 4 , there are observables $\left\{A_{s}\right\}$ and $\left\{B_{t}\right\}$ such that

$$
\left\langle\psi\left|A_{s} \otimes B_{t}\right| \psi\right\rangle=\left\langle u_{s} \mid v_{t}\right\rangle
$$

for all $s \in S$ and $t \in T$. Thus the strategy can be realized as a quantum strategy.

The maximization in Proposition 5 can be cast as a semidefinite program, which can be approximated to within an additive error of $\varepsilon$ in time polynomial in $|S|+|T|$ and in $\log (1 / \varepsilon)$. (See Ref. [8] for an introduction to semidefinite programming.)

It is trivial to write an expression similar to (5) for the classical value of an XOR game, viz.,

$$
\omega_{c}(G)-\tau(G)=\frac{1}{2} \max _{a(s), b(t)} \sum_{s, t} \pi(s, t)(V(0 \mid s, t)-V(1 \mid s, t)) a(s) b(t)
$$

where the maximum is over functions $a: S \rightarrow\{-1,+1\}$ and $b: T \rightarrow\{-1,+1\}$. This integer quadratic program is MAXSNP hard [1]. Unless $\mathrm{P}=\mathrm{NP}$, finding the quantum value of an $\mathrm{XOR}$ game is easier than finding the classical value.

\section{Upper bound for XOR games with weak classical strategies}

We now give two bounds for XOR games. We first consider the regime where the success probability of the best classical strategy is not much better than $\tau(G)$, the success probability of the trivial random strategy. In this case no quantum strategy can do significantly better. The bound will be expressed in terms of Grothendieck's constant [26].

Definition 6. Grothendieck's constant $K_{G}$ is the smallest number such that, for all integers $N \geq 2$ and all $N \times N$ real matrices $M$, if

$$
\left|\sum_{s, t} M(s, t) a_{s} b_{t}\right| \leq 1
$$


for all numbers $a_{1}, \ldots, a_{N}, b_{1}, \ldots, b_{N}$ in $[-1,1]$, then

$$
\left|\sum_{s, t} M(s, t)\left\langle u_{s} \mid v_{t}\right\rangle\right| \leq K_{G},
$$

for all unit vectors $\left|u_{1}\right\rangle, \ldots,\left|u_{N}\right\rangle,\left|v_{1}\right\rangle, \ldots,\left|v_{N}\right\rangle$ in $\mathbb{R}^{n}$ (for any choice of $n$ ).

(See also [20].) Grothendieck's constant is known to satisfy

$$
1.6769 \leq K_{G} \leq \frac{\pi}{2 \log (1+\sqrt{2})} \approx 1.7822,
$$

but the exact value is not known. The upper bound is due to Krivine [38] (who conjectures it is the exact value), and the lower bound is due to Davie [16] and, independently, Reeds [46] (see also [21]).

Theorem 7. Let $\mathrm{G}$ be an XOR game. Then

$$
\omega_{q}(G)-\tau(G) \leq K_{G}\left[\omega_{c}(G)-\tau(G)\right] .
$$

Proof. Suppose, without loss of generality, that $|S|=|T|$, and let $N=|S|=|T|$. Define an $N \times N$ matrix $M$ by

$$
M(s, t)=\frac{1}{2\left[\omega_{c}(G)-\tau(G)\right]} \pi(s, t)[V(0 \mid s, t)-V(1 \mid s, t)] .
$$

It follows that

$$
\left|\sum_{s, t} M(s, t) a_{s} b_{t}\right| \leq 1
$$

for all numbers $a_{1}, \ldots, a_{n}, b_{1}, \ldots, b_{n}$ in $[-1,1]$. By Proposition 5 , we have

$$
\omega_{q}(G)-\tau(G)=\left[\omega_{c}(G)-\tau(G)\right] \max _{\left\{\left|u_{s}\right\rangle\right\},\left\{\left|v_{t}\right\rangle\right\}} M(s, t)\left\langle u_{s} \mid v_{t}\right\rangle \leq K_{G}\left[\omega_{c}(G)-\tau(G)\right],
$$

which establishes the result.

For the $\mathrm{CHSH}$ game, we have $\tau(G)=1 / 2$ and

$$
\omega_{q}(G)-\tau(G)=\sqrt{2}\left[\omega_{c}(G)-\tau(G)\right]
$$

Games for which the ratio of $\omega_{q}(G)-\tau(G)$ to $\omega_{c}(G)-\tau(G)$ is greater than $\sqrt{2}$ can be constructed from the results in Ref. [21]. In particular, the smallest known game for which this ratio is larger than $\sqrt{2}$ has $|S|=|T|=20$.

\section{Upper bound for XOR games with strong classical strategies}

We now consider the regime where a classical strategy performs well, but not perfectly. For the Odd Cycle game of Section 3.2, we obtained

$$
\omega_{c}(G)=1-\frac{1}{2 n}
$$


and

$$
\omega_{q}(G) \geq \cos ^{2}(\pi / 4 n) \geq 1-(\pi / 4 n)^{2} ;
$$

the quantum strategy is quadratically better than the classical one in terms of its failure probability. In fact such a quadratic improvement is all that is possible for XOR games, as will be shown shortly in Theorem 8 .

In order to state and prove Theorem 8 , we first define a function $g:[0,1] \rightarrow[0,1]$ and two constants, $\gamma_{1}$ and $\gamma_{2}$. The function $g$ has the property that it is minimal subject to being concave and bounded below by $\sin ^{2}\left(\frac{\pi}{2} x\right)$. To determine $g$, consider the unique linear mapping $h(x)=\gamma_{1} x$ such that $h$ is the tangent line to $\sin ^{2}\left(\frac{\pi}{2} x\right)$ at some point $0<\gamma_{2}<1$. It is straightforward to show that $g(x)=h(x)$ for $x \leq \gamma_{2}$ and $g(x)=\sin ^{2}\left(\frac{\pi}{2} x\right)$ for $x>\gamma_{2}$. To determine the constants $\gamma_{1}$ and $\gamma_{2}$, note that the condition on $h$ and the fact that $\frac{\mathrm{d}}{\mathrm{d} x} \sin ^{2}\left(\frac{\pi}{2} x\right)=\frac{\pi}{2} \sin (\pi x)$ imply that

$$
\frac{\pi}{2} \sin \left(\pi \gamma_{2}\right)=\frac{\sin ^{2}\left(\frac{\pi}{2} \gamma_{2}\right)}{\gamma_{2}}=\gamma_{1}
$$

Theorem 8. Let $G$ be an XOR game with classical value $\omega_{c}(G)$. Then $\omega_{q}(G) \leq g\left(\omega_{c}(G)\right)$, where $g$ is as defined above, i.e.,

$$
\omega_{q}(G) \leq \begin{cases}\gamma_{1} \omega_{c}(G) & \text { if } \omega_{c}(G) \leq \gamma_{2} \\ \sin ^{2}\left(\frac{\pi}{2} \omega_{c}(G)\right) & \text { if } \omega_{c}(G)>\gamma_{2}\end{cases}
$$

where $\gamma_{1} \approx 1.1382$ and $\gamma_{2} \approx 0.74202$ are as defined above.

Proof. Consider an optimal quantum strategy and let

$$
\left\{\left|u_{s}\right\rangle: s \in S\right\},\left\{\left|v_{t}\right\rangle: t \in T\right\} \subset \mathbb{R}^{m}
$$

be the unit vectors associated with it, according to Proposition 5 . We use these vectors to define the following classical strategy:

1. Alice and Bob share a unit vector $|\lambda\rangle \in \mathbb{R}^{m}$, chosen uniformly at random.

2. When asked question $s$, Alice answers $a^{\prime}=\left[1+\operatorname{sign}\left(\left\langle\lambda \mid u_{s}\right\rangle\right)\right] / 2$.

3. When asked question $t$, Bob answers $b^{\prime}=\left[1+\operatorname{sign}\left(\left\langle\lambda \mid v_{t}\right\rangle\right)\right] / 2$.

Here the sign function is defined by $\operatorname{sign}(x)=+1$ if $x \geq 0$ and -1 otherwise.

Let us calculate the probability that $a^{\prime} \oplus b^{\prime}=1$. Introduce an azimuthal coordinate $\phi$ for $|\lambda\rangle$ in the plane spanned by $\left|u_{s}\right\rangle$ and $\left|v_{t}\right\rangle$, such that $\left|u_{s}\right\rangle$ has coordinate $\phi=0$ and $\left|v_{t}\right\rangle$ has coordinate $\phi=\theta_{s t} \equiv \cos ^{-1}\left\langle u_{s} \mid v_{t}\right\rangle \in[0, \pi]$. Then $\operatorname{sign}\left(\left\langle u_{s} \mid \lambda\right\rangle\right)=1$ for $\phi \in[-\pi / 2, \pi / 2]$ and -1 otherwise, while $\operatorname{sign}\left(\left\langle v_{t} \mid \lambda\right\rangle\right)=1$ for $\phi \in\left[\theta_{s t}-\pi / 2, \theta_{s t}+\pi / 2\right]$ and -1 otherwise. Because $|\lambda\rangle$ is distributed uniformly in $\mathbb{R}^{m}, \phi$ is distributed uniformly in $[0,2 \pi)$. The probability that $a \oplus b=1$ is then proportional to the measure of the subset of $[0,2 \pi)$ on which $\operatorname{sign}\left(\left\langle u_{s} \mid \lambda\right\rangle\right)=-\operatorname{sign}\left(\left\langle v_{t} \mid \lambda\right\rangle\right)$. In particular, $a^{\prime} \neq b^{\prime}$ when

$$
\phi \in\left[-\pi / 2, \theta_{s t}-\pi / 2\right) \cup\left[\pi / 2, \theta_{s t}+\pi / 2\right) .
$$

Therefore, on input $(s, t)$,

$$
\operatorname{Pr}\left[a^{\prime} \oplus b^{\prime}=1\right]=\frac{1}{\pi} \theta_{s t}
$$


Using the quantum strategy, the probability that $a \oplus b=1$ is given by

$$
\operatorname{Pr}[a \oplus b=1]=\frac{1}{2}\left(1-\left\langle u_{s} \mid v_{t}\right\rangle\right)=\sin ^{2}\left(\frac{1}{2} \theta_{s t}\right)
$$

so that

$$
\operatorname{Pr}[a \oplus b=1]=\sin ^{2}\left(\frac{\pi}{2} \operatorname{Pr}\left[a^{\prime} \oplus b^{\prime}=1\right]\right) \leq g\left(\operatorname{Pr}\left[a^{\prime} \oplus b^{\prime}=1\right]\right) .
$$

Similarly, it can be shown that

$$
\operatorname{Pr}[a \oplus b=0]=\sin ^{2}\left(\frac{\pi}{2} \operatorname{Pr}\left[a^{\prime} \oplus b^{\prime}=0\right]\right) \leq g\left(\operatorname{Pr}\left[a^{\prime} \oplus b^{\prime}=0\right]\right) .
$$

For each $(s, t) \in S \times T$, let $\omega_{\mathcal{c}}(s, t)$ and $\omega_{q}(s, t)$ be the probabilities of winning the game when using the classical and quantum strategies, respectively, given that question $(s, t)$ was asked. From the above, together with the concavity of $g$, it follows that $\omega_{q}(s, t) \leq g\left(\omega_{c}(s, t)\right)$. The overall probability of winning using the quantum strategy is

$$
\sum_{s, t} \pi(s, t) \omega_{q}(s, t) \leq \sum_{s, t} \pi(s, t) g\left(\omega_{c}(s, t)\right) \leq g\left(\sum_{s, t} \pi(s, t) \omega_{c}(s, t)\right) \leq g\left(\omega_{c}(G)\right),
$$

where we have again used the fact that $g$ is concave.

We emphasize that our means of defining the classical strategy in the above proof is not original; indeed we can trace the technique back to Grothendieck, who used it to establish the first upper bound on the constant that bears his name [26]. More recently, Goemans and Williamson used the same idea to derive randomized approximation algorithms for MAX-CUT and related problems [25].

One consequence of Theorem 8 is that the quantum strategy for the Odd Cycle game given in Section 3.2 is optimal.

Corollary 9. For $G$ being the Odd Cycle game, we have $\omega_{q}(G)=\cos ^{2}(\pi / 4 n)$.

\subsection{Bounds on entanglement for XOR games}

The final results we prove concern the amount of entanglement needed for Alice and Bob to play a given game optimally. With respect to this question, our results are restricted to XOR games. The following theorem follows immediately from the results of Section 5.3 .

Theorem 10. Let $G$ be an XOR game and let $m=\min (|S|,|T|)$. There exists an optimal strategy for Alice and Bob for $G$ in which they share a maximally-entangled state on $\lceil\mathrm{m} / 2\rceil$ qubits.

Unfortunately, even in this restricted setting of XOR games, the bound on the amount of entanglement provided by this theorem is still huge - the number of qubits shared by Alice and Bob is exponential in the sizes of their inputs.

However, if we are willing to settle for a slightly sub-optimal strategy, a polynomial number of shared qubits suffices. This fact follows from the Johnson-Lindenstrauss lemma [30], which we now state, following Ref. [15].

Lemma 11 (Johnson-Lindenstrauss). For $\varepsilon \in(0,1)$ and $n$ a positive integer, let $K$ be a positive integer such that

$$
K \geq 4\left(\varepsilon^{2} / 2-\varepsilon^{3} / 3\right)^{-1} \log n .
$$

Then for any set $V$ of $n$ points in $\mathbb{R}^{d}$, there is a mapping $f: \mathbb{R}^{d} \rightarrow \mathbb{R}^{K}$ such that for all $|u\rangle,|v\rangle \in V$,

$$
(1-\varepsilon) \||u\rangle-|v\rangle\left\|^{2} \leq\right\| f(|u\rangle)-f(|v\rangle)\left\|^{2} \leq(1+\varepsilon)\right\||u\rangle-|v\rangle \|^{2} .
$$


Theorem 12. Let $G=G(V, \pi)$ be an XOR game with quantum value $\omega_{q}(G)$. Let $0<\varepsilon<1 / 10$, and suppose $K$ is an even integer such that

$$
K \geq 4\left(\varepsilon^{2} / 2-\varepsilon^{3} / 3\right)^{-1} \log (|S|+|T|+1) .
$$

Then, if Alice and Bob share a maximally entangled state on $K / 2$ qubits, they can win with probability greater than $\omega_{q}(G)-\varepsilon$.

Proof. Let $M=|S|+|T|$ and $0<\varepsilon<1 / 10$. Let $\left\{\left|u_{s}\right\rangle: s \in S\right\}$ and $\left\{\left|v_{t}\right\rangle: t \in T\right\}$ be the vectors associated with the optimal quantum strategy according to Proposition 5, Let $f$ be the mapping obtained from the Johnson-Lindenstrauss Lemma to the $M+1$ points

$$
\left\{\left|u_{s}\right\rangle: s \in S\right\}, \quad\left\{\left|v_{t}\right\rangle: t \in T\right\}, \quad \text { and } 0 \text {. }
$$

Set

$$
\left|u_{s}^{\prime}\right\rangle=\frac{f\left(\left|u_{s}\right\rangle\right)-f(0)}{\| f\left(\left|u_{s}\right\rangle\right)-f(0) \|} \quad \text { and } \quad\left|v_{t}^{\prime}\right\rangle=\frac{f\left(\left|v_{t}\right\rangle\right)-f(0)}{\| f\left(\left|v_{t}\right\rangle\right)-f(0) \|} .
$$

for each $s \in S$ and $t \in T$. Because $\langle x \mid y\rangle=1-\|x-y\| / 2$ for real unit vectors $x$ and $y$, we have

$$
\left.\left|\left\langle u_{s}^{\prime} \mid v_{t}^{\prime}\right\rangle-\left\langle u_{s} \mid v_{t}\right\rangle\right|=\frac{1}{2}|\|| u_{s}^{\prime}\right\rangle-\left|v_{t}^{\prime}\right\rangle\|-\|\left|u_{s}\right\rangle-\left|v_{t}\right\rangle \| \mid
$$

A straightforward calculation based on the fact that

$$
\begin{aligned}
& \sqrt{1-\varepsilon} \leq \| f\left(\left|u_{s}\right\rangle\right)-f(0) \| \leq \sqrt{1+\varepsilon} \\
& \sqrt{1-\varepsilon} \leq \| f\left(\left|v_{t}\right\rangle\right)-f(0) \| \leq \sqrt{1+\varepsilon}
\end{aligned}
$$

and

$$
\sqrt{1-\varepsilon} \|\left|u_{s}\right\rangle-\left|v_{t}\right\rangle\|\leq\| f\left(\left|u_{s}\right\rangle\right)-f\left(\left|v_{t}\right\rangle\right)\|\leq \sqrt{1+\varepsilon}\|\left|u_{s}\right\rangle-\left|v_{t}\right\rangle \|
$$

proves that

$$
\left.\frac{1}{2}|\|| u_{s}^{\prime}\right\rangle-\left|v_{t}^{\prime}\right\rangle\|-\|\left|u_{s}\right\rangle-\left|v_{t}\right\rangle \| \mid<2 \varepsilon
$$

We note that these vectors can be realized as a quantum strategy by Theorem 4 . It follows that the difference in the probability of winning using this strategy instead of the optimal one is

$$
\begin{aligned}
\frac{1}{2} \sum_{s, t} \pi(s, t)[ & V(0 \mid s, t)-V(1 \mid s, t)]\left(\left\langle u_{s} \mid v_{t}\right\rangle-\left\langle u_{s}^{\prime} \mid v_{t}^{\prime}\right\rangle\right) \\
\leq & \frac{1}{2} \sum_{s, t} \pi(s, t)\left|\left\langle u_{s} \mid v_{t}\right\rangle-\left\langle u_{s}^{\prime} \mid v_{t}^{\prime}\right\rangle\right| \leq \epsilon \sum_{s, t} \pi(s, t)=\varepsilon .
\end{aligned}
$$

Hence Alice and Bob win using this strategy with probability greater than $\omega_{q}(G)-\varepsilon$.

Oded Regev has described to us an improved form of this theorem where $K$ has no dependence on $|S|$ and $|T|$. 


\subsection{Further connections with multi-prover interactive proof systems}

One motive for considering upper bounds on the quantum values of games in general is due to their connections with multi-prover interactive proof systems. For example, recall that the Odd Cycle game can be regarded as a simple proof system for the two-colorability of odd cycles-for which the correct response of the verifier is to reject. Although this is valid as a classical twoprover interactive proof system, if the quantum value of the game were one (or exponentially close to one) then it would not be valid as a quantum proof system. The upper bound on the value of the Odd Cycle game proved in this section (Corollary 9 ) implies that it is a valid quantum proof system, and with a polynomial number of repetitions 2 , the probability of the verifier incorrectly accepting can be made arbitrarily close to zero. For any one-round two-prover quantum interactive proof system, the soundness condition will correspond to a nontrivial upper bound of the quantum value of a nonlocality game. Therefore upper bounds are important tools for analyzing such proof systems.

Regarding upper bounds on entanglement required by an optimal quantum strategy, we note that results in [36] imply that if a polynomial upper bound can be established then MIP* $^{*}[2] \subseteq$ NEXP. This indicates that upper bounds on entanglement are also relevant for analyzing such proof systems.

Definition 13. For $0 \leq s<c \leq 1$, let $\oplus \operatorname{MIP}_{c, s}[2]$ denote the class of all languages $L$ recognized by classical two-prover interactive proof systems of the following form:

- They operate in one round, each prover sends a single bit in response to the verifier's question, and the verifier's decision is a function of the parity of those two bits.

- If $x \notin L$ then, whatever strategy Alice and Bob follow, the Prover's acceptance probability is at most $s$ (the soundness probability).

- If $x \in L$ then there exists a strategy for Alice and Bob for which the Prover's acceptance probability is at least $c$ (the completeness probability).

Definition 14. For $0 \leq s<c \leq 1$, let $\oplus \mathrm{MIP}_{c, s}^{*}[2]$ denote the class corresponding to the previous definition, where all communication remains classical, but where the provers may share prior quantum entanglement.

The following result is implicit in the work of Håstad [27], based on the application of methods described in [6].

Theorem 15. For all $\varepsilon \in(0,1 / 16)$, if $s=11 / 16+\varepsilon$ and $c=12 / 16$ then $\oplus \operatorname{MIP}_{c, s}[2]=\operatorname{NEXP}$.

Sketch of Proof. We refer the reader to [6, 27] for all detailed information about probabilistically checkable proof systems (PCPs). Let $\mathrm{PCP}_{c, s}[r, k]$ denote the class of languages recognized by PCPs that makes $k$ queries on the basis of $r$ random bits, and have completeness and soundness probabilities $c$ and $s$ respectively. That is, a verifier can query $k$ bits of a purported proof, selected on the basis of $r$ random bits, and makes a determination of language membership on the basis of those $k$ values. A language $L$ is in $\mathrm{PCP}_{c, s}[r, k]$ if: (a) for all $x \in L$, there exists a proof for which the verifier's acceptance probability is at least $c$; and (b) for all $x \notin L$, the verifier's acceptance never exceeds s. Håstad [27] essentially shows that, for all $\varepsilon>0$, if $s=11 / 16+\varepsilon$, and $c=12 / 16$ then

\footnotetext{
${ }^{2}$ In the absence of a quantum analogue of Raz's Parallel Repetition Theorem [45], the repetitions can be applied sequentially.
} 
$\mathrm{PCP}_{c, s}[\mathrm{O}(\log n), 2]=\mathrm{NP}$ using PCPs where the verifier's determination is based on the XOR of the two queried bits. This can be scaled up one exponential in $n$ along the lines discussed in [6] to yield $\mathrm{PCP}_{c, s}\left[n^{\mathrm{O}(1)}, 2\right]=\mathrm{NEXP}$ with the same XOR property. Moreover, the proof system has the feature that, if each possible pair of queries is taken as an edge of a graph then the resulting graph is bipartite. This means that the PCP can be converted into a two-prover interactive proof system with the same completeness and soundness probabilities ( $c$ and $s$ ) as follows. The verifier randomly chooses an edge, just as in the PCP, and sends one query to Alice and one to Bob, according to the bipartite structure of the graph.

An obvious question is: Do there exist $c$ and $s$ (with $0 \leq s<c \leq 1$ ) such that $\oplus \operatorname{MIP}_{c, s}^{*}[2]=$ NEXP? The answer is that this cannot be the case unless EXP $=$ NEXP, following from the discussion in Section 5.3 .

Corollary 16. For all $s$ and $c$ such that $0 \leq s<c \leq 1, \oplus \mathrm{MIP}_{c, s}^{*}[2] \subseteq \mathrm{EXP}$.

\section{Acknowledgments}

This work was primarily done while RC and JW were at the University of Calgary and BT was at the California Institute of Technology, and was partially supported by Canada's NSERC, MITACS, PIMS, and CIFAR; the U.S. National Science Foundation under Grant No. EIA-0086038; and Alberta's iCORE.

We would like to thank Andris Ambainis, Dave Bacon, Anne Broadbent, Andrew Doherty, Nicolas Gisin, David Mermin, Asher Peres, John Preskill, Oded Regev, Madhu Sudan, and Alain Tapp for helpful discussions, Steven Finch for providing us with copies of Refs. [16] and [46], and the anonymous referees for several helpful comments and corrections.

\section{References}

[1] N. Alon and A. Naor. Approximating the cut-norm via Grothendieck's inequality. In Proceedings of the Thirty-Sixth Annual ACM Symposium on Theory of Computing, 2004.

[2] A. Ambainis. A new protocol and lower bounds for quantum coin flipping. In Proceedings of the Thirty-Third Annual ACM Symposium on Theory of Computing, pages 134-142, 2001.

[3] P. K. Aravind. The magic squares and Bell's theorem. Manuscript, 2002. Available as arXiv.org e-Print quant-ph/0206070.

[4] L. Babai, L. Fortnow, and C. Lund. Non-deterministic exponential time has two-prover interactive protocols. Computational Complexity, 1(1):3-40, 1991.

[5] J. Bell. On the Einstein-Podolsky-Rosen paradox. Physics, 1(3):195-200, 1964.

[6] M. Bellare, O. Goldreich, and M. Sudan. Free bits, PCPs, and non-approximability - towards tight results. SIAM Journal on Computing, 27(3):804-915, 1998.

[7] M. Ben-Or, S. Goldwasser, J. Kilian, and A. Wigderson. Multi-prover interactive proofs: how to remove intractability assumptions. In Proceedings of the 20th Annual ACM Symposium on Theory of Computing, pages 113-131, 1988.

[8] S. Boyd and L. Vandenberghe. Convex Optimization. Cambridge University Press, 2004. 
[9] G. Brassard, R. Cleve, and A. Tapp. Cost of exactly simulating quantum entanglement with classical communication. Physical Review Letters, 83(9):1874-1877, 1999.

[10] S. Braunstein and C. Caves. Wringing out better Bell inequalities. Annals of Physics, 202:22-56, 1990.

[11] H. Buhrman, R. Cleve, and A. Wigderson. Quantum vs. classical communication and computation. In Proceedings of the Thirtieth Annual ACM Symposium on Theory of Computing, pages 63-68, 1998.

[12] J. Cai, A. Condon, and R. Lipton. On bounded round multi-prover interactive proof systems. In Proceedings of the Fifth Annual Conference on Structure in Complexity Theory, pages 45-54, 1990.

[13] J. Cai, A. Condon, and R. Lipton. PSPACE is provable by two provers in one round. Journal of Computer and System Sciences, 48(1):183-193, 1994.

[14] J. F. Clauser, M. A. Horne, A. Shimony, and R. A. Holt. Proposed experiment to test local hidden-variable theories. Physical Review Letters, 23(15):880-884, 1969.

[15] S. Dasgupta and A. Gupta. An elementary proof of the Johnson-Lindenstrauss lemma. Technical Report TR-99-006, International Computer Science Institute, Berkeley, California, 1999.

[16] A. M. Davie. Lower bound for $K_{G}$. Unpublished note, 1984.

[17] A. Doherty, Y. Liang, B. Toner, and S. Wehner. The quantum moment problem and bounds on entangled multi-prover games. In Proceedings of the 23rd Annual IEEE Conference on Computational Complexity, pages 199-210, 2008.

[18] U. Feige. On the success probability of two provers in one-round proof systems. In Proceedings of the Sixth Annual Conference on Structure in Complexity Theory, pages 116-123, 1991.

[19] U. Feige and L. Lovász. Two-prover one-round proof systems: their power and their problems. In Proceedings of the Twenty-Fourth Annual ACM Symposium on Theory of Computing, pages 733-744, 1992.

[20] S. R. Finch. Mathematical Constants. Cambridge University Press, 2003.

[21] P. Fishburn and J. Reeds. Bell inequalities, Grothendieck's constant, and root two. SIAM Journal on Discrete Mathematics, 7:48-56, 1994.

[22] L. Fortnow, J. Rompel, and M. Sipser. On the power of multi-prover interactive protocols. Theoretical Computer Science, 134:545-557, 1994.

[23] P. Frankl and V. Rodl. Forbidden intersections. Transactions of the American Mathematical Society, 300(1):259-286, 1987.

[24] V. Galliard, A. Tapp, and S. Wolf. The impossibility of pseudo-telepathy without quantum entanglement. Available as arXiv.org e-Print quant-ph/0211011, 2002.

[25] M. X. Goemans and D. P. Williamson. Improved approximation algorithms for maximum cut and satisfiability problems using semidefinite programming. Journal of the ACM, 42:11151145, 1995. 
[26] A. Grothendieck. Résumé de la théorie métrique des produits tensoriels topologiques. Boletim Da Sociedade de Matemática de São Paulo, 8:1-79, 1953.

[27] J. Håstad. Some optimal inapproximability results. Journal of the ACM, 48(4):798-859, 2001.

[28] P. Heywood and M. L. G. Redhead. Nonlocality and the Kochen-Specker paradox. Foundations of Physics, 13:481-499, 1983.

[29] T. Ito, H. Kobayashi, D. Preda, X. Sun, and A. Yao. Generalized Tsirelson inequalities, commuting-operator provers, and multi-prover interactive proof systems. In Proceedings of the 2008 IEEE 23rd Annual Conference on Computational Complexity, pages 187-198, 2008.

[30] W. Johnson and J. Lindenstrauss. Extensions of Lipschitz maps into a Hilbert space. Contemporary Mathematics, 26:189-206, 1984.

[31] P. Kaye, R. Laflamme, and M. Mosca. An Introduction to Quantum Computing. Oxford University Press, 2007.

[32] J. Kempe, H. Kobayashi, K. Matsumoto, B. Toner, and T. Vidick. Entangled games are hard to approximate. In Proceedings of the 49th Annual IEEE Symposium on Foundations of Computer Science, 2008.

[33] J. Kempe, O. Regev, and B. Toner. Unique games with entangled provers are easy. In Proceedings of the 2008 49th Annual IEEE Symposium on Foundations of Computer Science, pages 457-466, 2008.

[34] L. A. Khalfin and B. S. (Tsirelson) Tsirel'son. Quantum and quasi-classical analogs of Bell inequalities. In P. Lahti and P. Mittelstaedt, editors, Symposium on the Foundations of Modern Physics, pages 441-460. World Scientific, 1985.

[35] A. Kitaev, A. Shen, and M. Vyalyi. Classical and Quantum Computation, volume 47 of Graduate Studies in Mathematics. American Mathematical Society, 2002.

[36] H. Kobayashi and K. Matsumoto. Quantum multi-prover interactive proof systems with limited prior entanglement. Journal of Computer and System Sciences, 66(3), 2003.

[37] S. Kochen and E. P. Specker. The problem of hidden variables in quantum mechanics. Journal of Mathematics and Mechanics, 17:59-87, 1967.

[38] J. L. Krivine. Constantes de Grothendieck et fonctions de type positif sur les sphères. Advances in Mathematics, 31:16-30, 1979.

[39] D. Lapidot and A. Shamir. Fully parallelized multi prover protocols for NEXP-time. In Proceedings of the 32nd Annual IEEE Symposium on Foundations of Computer Science, pages 13-18, 1991.

[40] D. Leung, B. Toner, and J. Watrous. Coherent state exchange in multi-prover quantum interactive proof systems. Available as arXiv.org e-Print 0804.4118, 2008.

[41] N. D. Mermin. Simple unified form for no-hidden variables theorems. Physical Review Letters, 65(27):3373-3376, 1990. 
[42] N. D. Mermin. Hidden variables and the two theorems of John Bell. Reviews of Modern Physics, 65(3):803-815, 1993.

[43] M. A. Nielsen and I. L. Chuang. Quantum Computation and Quantum Information. Cambridge University Press, 2000.

[44] A. Peres. Quantum Theory: Concepts and Methods. Kluwer, 1993.

[45] R. Raz. A parallel repetition theorem. SIAM Journal on Computing, 27(3):763-803, 1998.

[46] J. A. Reeds. A new lower bound on the real Grothendieck constant. Unpublished note, 1991.

[47] B. S. (Tsirelson) Cirel'son. Quantum generalizations of Bell's inequality. Letters in Mathematical Physics, 4(2):93-100, 1980.

[48] B. S. (Tsirelson) Tsirel'son. Quantum analogues of the Bell inequalities: The case of two spatially separated domains. Journal of Soviet Mathematics, 36:557-570, 1987.

[49] L. Vaidman. Tests of Bell inequalities. Manuscript, 2001. Available as arXiv.org e-Print quant-ph/0107057. 